\title{
Some evidence of ground power enhancements at frequencies of global magnetospheric modes at low latitude
}

\author{
P. Francia, U. Villante \\ Dipartimento di Fisica, Universita' dell'Aquila, I-L'Aquila, Italy
}

Received: 29 November 1995/Revised: 17 July 1996/Accepted: 22 July 1996

\begin{abstract}
A statistical analysis of the power spectra of the geomagnetic field components $H$ and $D$ for periods ranging between $3 \mathrm{~min}$ and $1 \mathrm{~h}$ was conducted at a lowlatitude observatory (L'Aquila, $L=1.6$ ) at the minimum and maximum of the solar cycle. For both components, during daytime intervals, we found evidence of power enhancements at frequencies predicted for global modes of the Earth's magnetosphere and occasionally observed at auroral latitudes in the $F$-region drift velocities (approximately at 1.3, 1.9, 2.6, and $3.4 \mathrm{mHz}$ ). Nighttime observations reveal a relative low frequency $H$ enhancement associated with the bay occurrence together with a peak in the $H / D$ power ratio which sharply emerges at $1.2 \mathrm{mHz}$ in the premidnight sector. The strong similarity between solar minimum and maximum suggests that these modes can be considered permanent magnetospheric features. A separate analysis on a two-month interval shows that the observed spectral characteristics are amplified by conditions of high-velocity solar wind.
\end{abstract}

\section{Introduction}

In the last several years a number of investigations reported clear evidence at auroral latitudes of long-period ULF waves in the F-region drift velocities with peaks of power at selected frequencies of the order of 1.3, 1.9, 2.6, and $3.4 \mathrm{mHz}$ (Ruohoniemi et al., 1991; Samson et al., 1992; Walker et al., 1992). Samson et al. (1992) and Ziesolleck and McDiarmid (1994) showed that these peaks of power can also be identified in the simultaneous fluctuations of the local ground magnetic field. These events typically are of long duration, have stable frequencies (within 10\%) and most frequently occur near local midnight and in the very early morning, although Ziesolleck and McDiarmid

Correspondence to: P. Francia
(1994) recently proposed similar observations in the dayside hemisphere. Following the original suggestion by Kivelson and Southwood (1985), these oscillations are currently interpreted in terms of field line resonances excited by compressional modes of the magnetospheric cavity (Samson et al., 1992; Walker et al., 1992). For example, Walker et al. (1992) showed that the observed postmidnight oscillations at 1.3 and $2.7 \mathrm{mHz}$ were localized on field lines at $L=14$ and 11.8 , respectively.

At low latitudes, interesting examples of similar lowfrequency signals have been so far detected only across the Australia Wide Array of Geomagnetic Stations (AWAGS, $1.1<L<2.3$; Ziesolleck and Chamalaun, 1993) throughout the dayside magnetosphere, and the observed ground signatures have been considered consistent with features expected for global compressional modes or large-scale cavity resonances trapped in the magnetosphere. According to theoretical models (Samson and Rankin, 1994), within the magnetosphere low-frequency cavity modes could be excited by several phenomena, such as pressure pulses from the solar wind, Kelvin-Helmholtz instabilities on the magnetopause, or transient dayside reconnection. They are modeled as fast compressive waves propagating antisunward through the field lines and reflected between the surface of inner turning points and outer boundaries such as the magnetopause or the bow shock; the evanescent barrier penetration would be responsible for ground observations. Harrold and Samson (1992), who considered the Earth's bow shock as an outer boundary, proposed discrete wave modes at 1.3, 1.9, 2.5, 3.4, and $4.2 \mathrm{mHz}$, which were well consistent with the experimental observations. They also suggested a lower-frequency surface wave at approximately $0.4 \mathrm{mHz}$, corresponding to the eigenmode of the Kelvin-Helmholtz instability, and a slow-mode wave, at approximately $0.7 \mathrm{mHz}$, reflecting between the bow shock and the low-latitude boundary layer.

In this present investigation we analyzed the power spectra (3-60 min) of the geomagnetic field components ( $H$ and $D)$ at L'Aquila $(L=1.6)$, and found, both at solar minimum (1985-1986) and maximum (1989-1990) and for 
both components, statistical evidence in the daytime spectra of power enhancements at approximately the same frequencies detected during nighttime intervals at higher latitude mostly in the F-region drift velocities. We also examined the ratio $W$ of the $H$ and $D$ spectral densities, which may be useful to distinguish between different modes. Indeed, far from the resonance frequency, ionospheric effects should be negligible for the expected fastmode oscillations (Kivelson and Southwood, 1988) and ground $W$ enhancements might be considered indicative of magnetospheric compressive perturbations which mostly occur in the meridian plane. In this sense, the results of our analysis do not reveal any evidence of $W$ enhancements during daytime intervals; conversely, nighttime observations show (associated with bays) a lowfrequency $W$ enhancement between approximately 0.5 and $2 \mathrm{mHz}$ and peak values of the $W$ parameter at $1.2 \mathrm{mHz}$.

\section{Experimental observations}

We adopted as original data the 1-min measurements of the horizontal component $H$ and declination $D$ (expressed in nT) measured during 1985-1986 (solar minimum) and 1989-90 (solar maximum) at L'Aquila Geomagnetic Observatory $(L=1.6 ; \mathrm{LT}=\mathrm{UT}+1)$ run by the Istituto Nazionale di Geofisica. As described by Meloni et al. (1984), the instrument basically consists of a proton precession magnetometer equipped with two Helmoltz coils and provides confident measurements of the geomagnetic field components in a wide frequency range. We only considered days with the local $K$ index smaller than 5 in any 3-h interval and without SSC. For each day we computed the power spectra of the geomagnetic field components, $P_{H}(f)$ and $P_{D}(f)$, over four consecutive 3-h intervals for daytime and nighttime intervals, respectively. The power spectra have been computed by means of the maximum entropy method at order $m=30$ of the prediction error filter (Vellante and Villante, 1984) for periods ranging between 3 and $60 \mathrm{~min}$. Average daytime (nighttime) spectra come from 1658 (1694) individual spectra at solar minimum and 2095 (2152) individual spectra at solar maximum. Average values of the solar-wind velocity and dynamic pressure are, respectively, $462 \mathrm{~km} \mathrm{~s}^{-1}$ and $3.2 \mathrm{nPa}$ at solar minimum, and $439 \mathrm{~km} \mathrm{~s}^{-1}$ and $2.6 \mathrm{nPa}$ at solar maximum.

In Fig. 1 we show the average power spectra of the $H$ and $D$ components. As can be seen, superimposed on the general power decrease with the increasing frequency, the statistical results show several power enhancements which typically have amplitude greater than (or comparable with) the statistical uncertainty for a $95 \%$ confidence interval, and tend to occur close to the frequencies of the long period fluctuations detected at higher latitude and interpreted in terms of magnetospheric cavity modes (arrows in Fig. 1 identify the frequencies predicted by Harrold and Samson, 1992). During daytime intervals (Fig. 1a) at solar minimum, the $H$ component has a higher energy content than $D$, and simultaneous $H$ and $D$ power enhancements emerge at approximately 1.4, 1.9, 2.5, and (with less evidence) at 3.6 and $4.2 \mathrm{mHz}$. At solar maximum, the $D$ component appears more energetic than $H$ and, for both components, the level of power at periods smaller than $10 \mathrm{~min}(1.7 \mathrm{mHz})$ shows a faster decrease with increasing frequency than at solar minimum. In this case the clearest power enhancement emerges in both the spectra at $1.9 \mathrm{mHz}$, and some enhanced activity is also detected at approximately $0.7 \mathrm{mHz}$.

Nighttime spectra (Fig. 1b) reveal a much greater energy content in the $H$ component between approximately 0.5 and $2 \mathrm{mHz}$. No clear simultaneous enhancements emerge in this case in the $H$ and $D$ power spectra; they rather show, more explicitly at solar minimum, some evidence for a power enhancement in the $H$ component at approximately $1.2 \mathrm{mHz}$, while other $H$ enhancements can be tentatively identified at approximately 0.7 and $1.9 \mathrm{mHz}$.

In order to examine more carefully the relative importance of the geomagnetic field components, we also focused our attention on the ratio of the spectral densities $W(f)=P_{H}(f) / P_{D}(f)$. As it can be seen, during daytime intervals (Fig. 2a) and for both phases of the solar cycle, $W$ attains an approximately constant value (which is appreciably smaller at solar maximum) for a major portion of the frequency range (approximately above $0.8 \mathrm{mHz}$ ) and reaches higher values at the lowest frequencies $(T>30 \mathrm{~min})$.

Nighttime observations (Fig. 2b) are more interesting in that they confirm the low-frequency relative increase in the $H$ component between 0.5 and $2 \mathrm{mHz}$, and show maximum $W$ values at approximately $1.2 \mathrm{mHz}$ at both phases of the solar cycle. Separate analysis of the $W$ behavior for different nighttime intervals (Fig. 3) shows a very interesting general correspondence between solar minimum and maximum observations in all LT sectors. They reveal that the low-frequency $W$ enhancement appears much more clearly in the premidnight sectors 18-21 and, more particularly, 21-24 LT, while it progressively disappears after local midnight. In addition, a comparison among the premidnight sectors 21-24 LT reveals that this low-frequency $W$ enhancement more explicitly emerges during 1985-1986, suggesting a higher average relative low-frequency power in the $H$ fluctuations at solar minimum. The peak value of the $W$ parameter, in the 18-21LT sector, is of the order of 1.8 at both phases of the solar cycle and emerges at frequencies of the order of 0.8 (solar minimum) and $1.1 \mathrm{mHz}$ (solar maximum). In the 21-24LT sectors, the $W$ peak emerges more sharply: at solar maximum it maintains approximately the same frequency and same relative magnitude as in the preceding LT sector; at solar minimum, low-frequency $W$ values become higher than 2 and the $W$ peak emerges more sharply at approximately $1.2 \mathrm{mHz}$.

The low-frequency $W$ enhancement and the associated peak values might be related to the bay occurrence which at our latitudes typically occur in the premidnight sector and mostly perturb the $H$ component (Chapman and Bartels, 1951; Nishida, 1978; Francia et al., 1995). This point is made clear in Fig. 4, in which we show the observations corresponding to a typical bay. As it can be seen, the bay occurrence is associated in this particular 

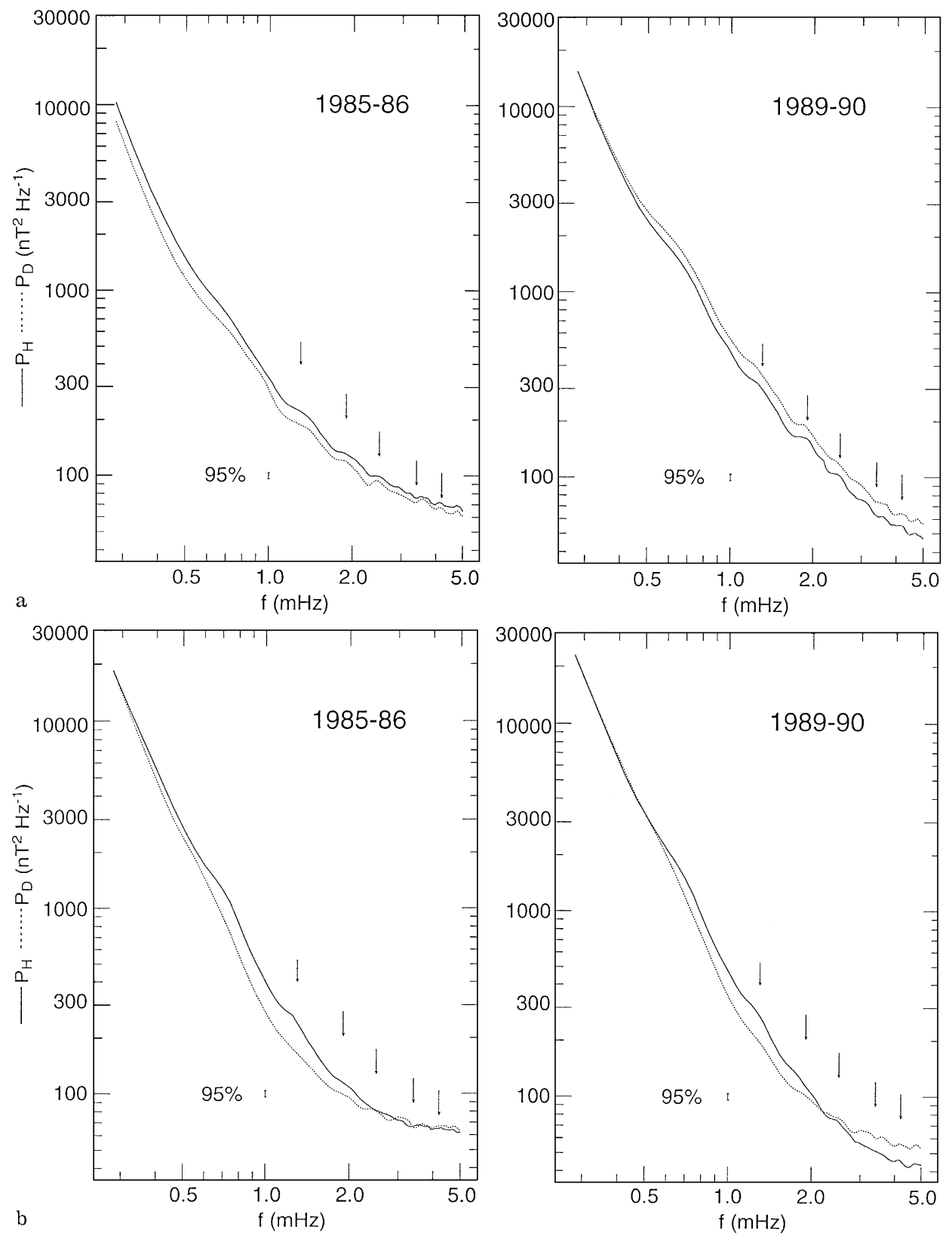

Fig. 1a, b. Power spectra $(\log -\log$ scale) of the horizontal components $H$ and $D$. Arrows identify the cavity-mode frequencies predicted by Harrold and Samson (1992). Bar at $1 \mathrm{mHz}$ corresponds to the $95 \%$ confidence interval. a Daytime spectra; b nighttime spectra

case with a power level that at frequencies smaller than $2.5 \mathrm{mHz}$ is much higher in the $H$ component; in addition, we found clear evidence for a further $H$ enhancement that, for this event, is identified at approximately $1.3-1.4 \mathrm{mHz}$.

As previously remarked, the low-latitude pulsation activity, as well as the onset of global magnetospheric modes, might be related to external parameters such as the solar-wind velocity and dynamic pressure. The statistical results show that, as already discussed, several aspects of the geomagnetic field fluctuations more clearly emerge at the solar minimum when both the average solar-wind velocity and dynamic pressure are higher. We then found it interesting to conduct a separate analysis for a two-month interval (July-August 1985) during which the average solar-wind velocity was very high $\left(511 \mathrm{~km} \mathrm{~s}^{-1}\right)$. The results of this analysis (Fig. 5) reinforce the suggestions of Fig. 1a: indeed higher solar-wind velocities provide a relative higher level of geomagnetic field fluctuations in the $H$ component together with a more important contribution of power at periods smaller than $10 \mathrm{~min}(1.7 \mathrm{mHz})$. In particular, when compared with Fig. 1a, the results of Fig. 5a show during daytime intervals a much larger separation between $H$ and $D$ spectra in the whole frequency range with the $H$ spectrum which is significantly higher than the average spectrum for the whole period 1985-1986. It is also very interesting to note that in Fig. 5a all the discussed spectral enhancements emerge more sharply at the expected frequencies than for the whole of 1985-1986. Similarly, during nighttime intervals (Fig. 5b), the $W$ ratio is remarkably higher 

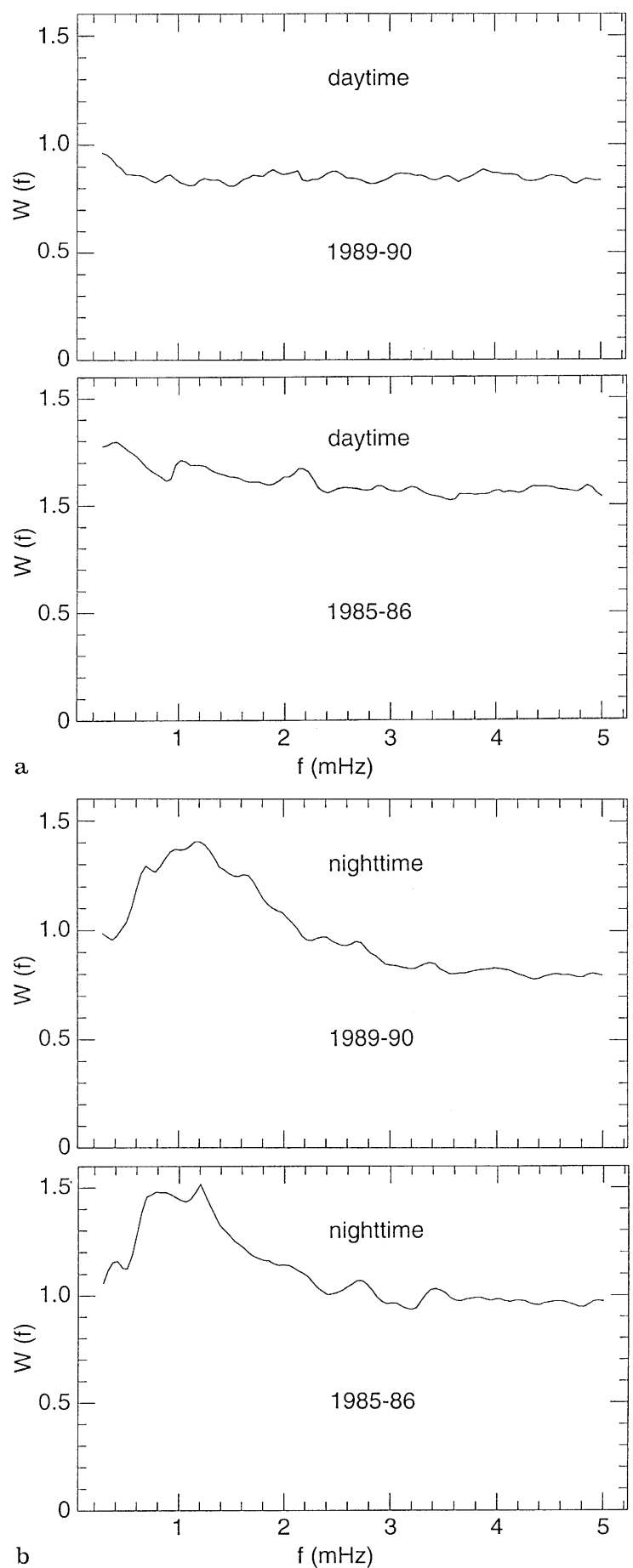

Fig. 2a, b. The $W$ ratio between the $H$ and $D$ spectral densities; a daytime intervals; $\mathbf{b}$ nighttime intervals

than for the whole of 1985-1986 (dotted line) and $W$ peaks appear much more clearly within few percent of all the predicted frequencies (namely 1.3, 1.9, 2.5, 3.4, and $4.2 \mathrm{mHz}$; Harrold and Samson, 1992), including the lowest frequency modes at 0.4 and $0.7 \mathrm{mHz}$. Note also that the additional enhancement at $2.2 \mathrm{mHz}$ might find correspondence in the experimental observations by Ziesolleck and McDiarmid (1994).
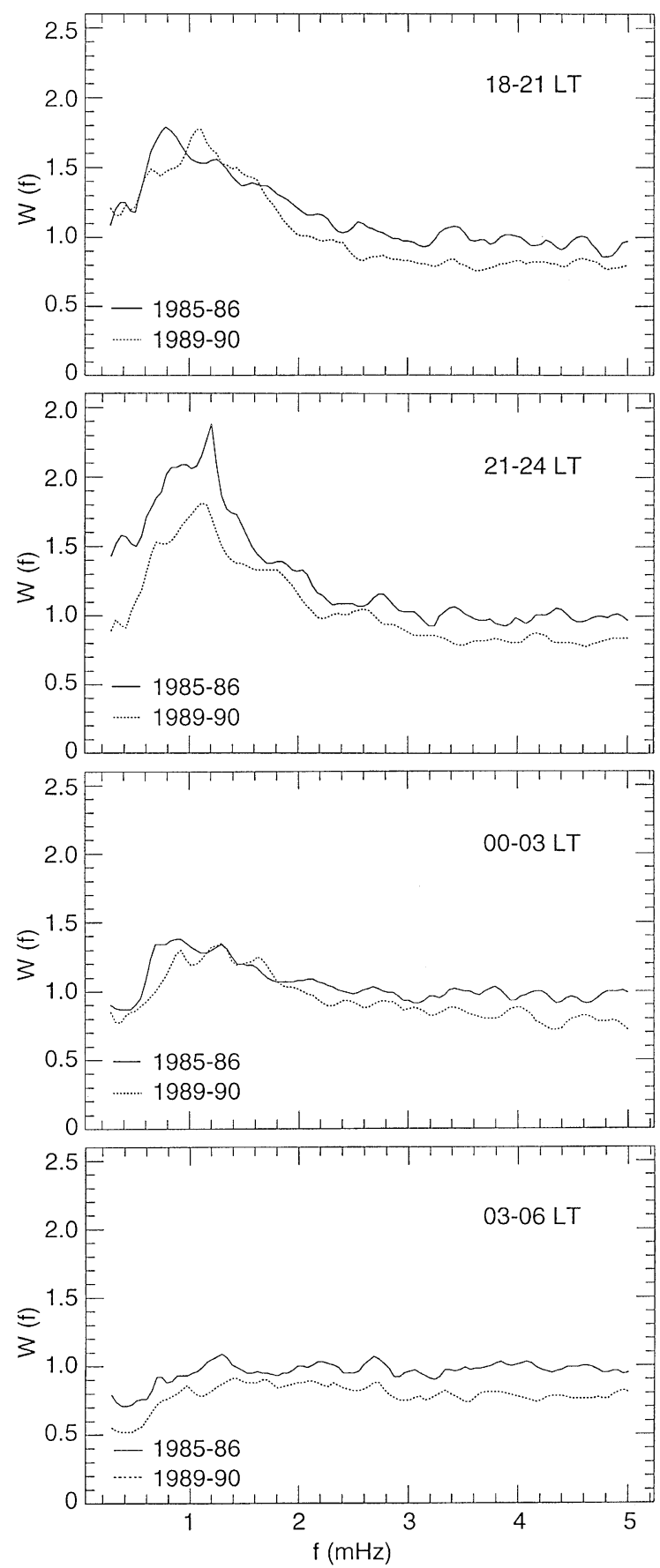

Fig. 3. The $W$ ratio between the $H$ and $D$ spectral densities for the four 3 -h nighttime intervals at minimum (solid line) and maximum (dotted line) of the solar cycle

\section{Summary and discussion}

In the present investigation we analyzed the power enhancements in the average spectra of the geomagnetic field fluctuations between 3 and $60 \mathrm{~min}$ at the minimum and maximum of the solar activity at a low-latitude observatory, and carefully examined the relative magnitude of $H$ and $D$ fluctuations. On this statistical basis, and after 

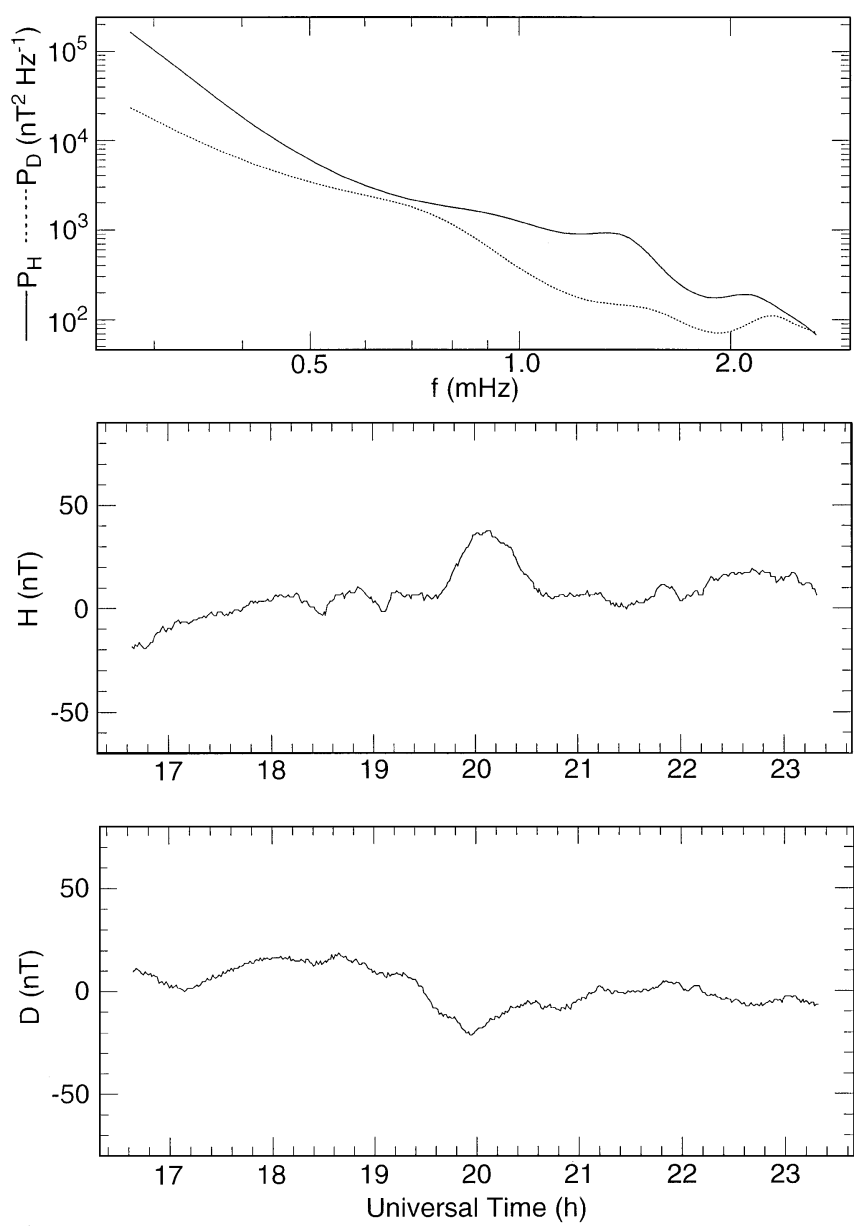

Fig. 4. Power spectra and time-series for 7 June 1985 event (20-23 LT)

removing the major effects of an active magnetosphere, we found a general correspondence between observations performed at the minimum and maximum of the solar activity which makes us sure that the basic conclusions of the present investigation represent permanent features of the low-latitude geomagnetic field observations. Nevertheless, it is interesting to underline that some important aspects of the experimental observations emerge more clearly at solar minimum when the average solar-wind velocity and dynamic pressure are greater.

From the results of the present investigation we can draw the following conclusions:

a Daytime average power spectra show in both components several power enhancements at frequencies approximately corresponding to the magnetospheric cavity or waveguide-mode frequencies predicted by Harrold and Samson (1992), who proposed the bow shock as external boundary and speculated discrete modes at 1.3, 1.9, 2.5, 3.4 , and $4.2 \mathrm{mHz}$. At these frequencies, waves observed at auroral latitudes in the F-region drift velocities (Ruohoniemi et al., 1991; Samson et al., 1992; Walker et al., 1992) were interpreted in terms of shear Alfvén modes on dipole-like field lines with resonant frequencies matching the magnetospheric cavity-mode eigenfrequen-
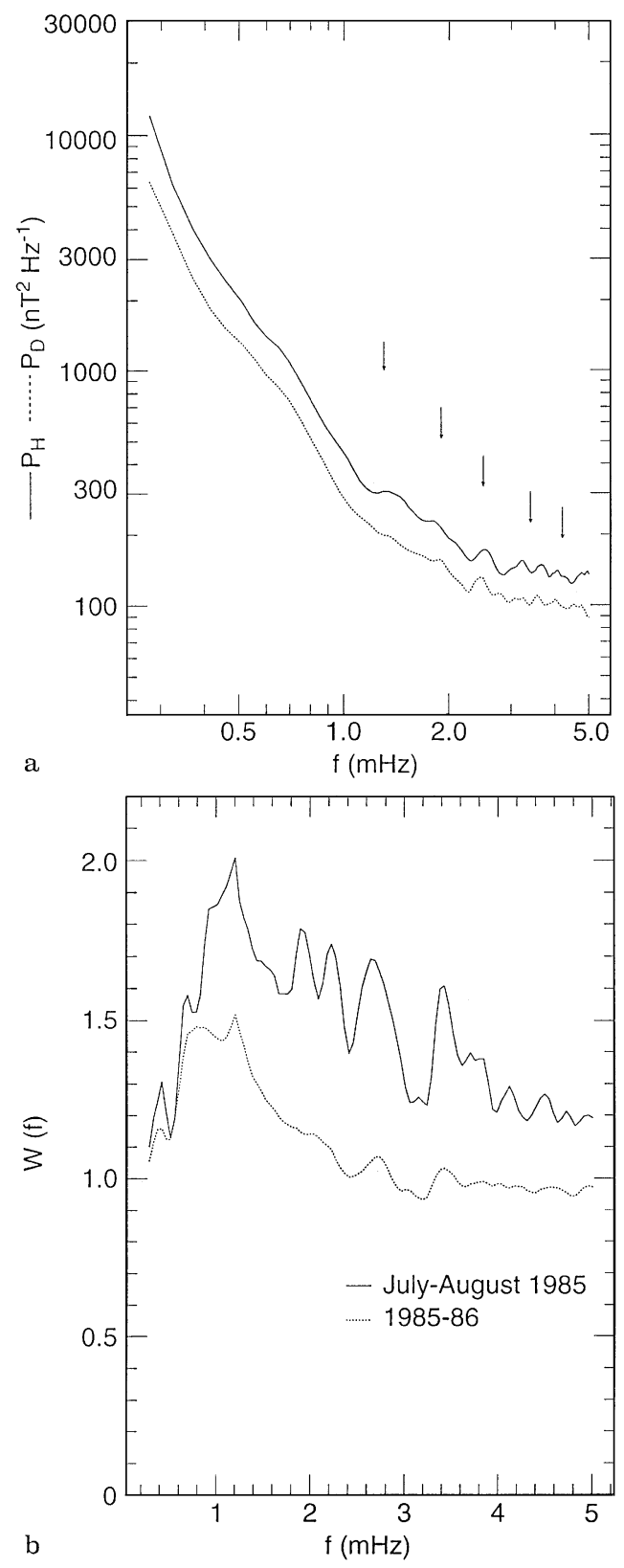

Fig. 5. a Daytime power spectra $(\log -\log$ scale) of the horizontal components $H$ and $D$ for the two-month interval July-August 1985; b the $W$ ratio between the $H$ and $D$ nighttime spectral densities for the two-month interval July-August 1985 (solid line) and for the whole interval 1985-1986 (dotted line)

cies. Our statistical results suggest that power enhancements might be solar-cycle-independent features of the Earth's magnetosphere which can be observed at low latitude during daytime intervals in both the geomagnetic field components. The correspondence with auroral observations at approximately the same frequencies tentatively suggests the interpretation of our experimental observations in terms of global magnetospheric modes. According to theoretical models (Kivelson, 1995, and references therein), far from the local resonance frequency, the compressional modes should mostly be detected along 
the $H$ component. Conversely, our results during daytime intervals do not suggest a dominant oscillation along the $H$ component at selected frequencies. Nevertheless, our results are consistent with the low-latitude observations conducted by Ziesolleck and Chamalaun (1993), who found magnetic signatures for similar fluctuations in both horizontal components and interpreted their observations in terms of global magnetospheric modes. They speculated indeed that the physical characteristics of the daytime ionosphere may well lead to a rotation of the polarization axis of the expected compressional mode (see also Itonaga and Kitamura, 1993) which may provide a significant ground signature also on the east-west component. Also, we found some statistical evidence for a lower frequency enhancement at $0.7 \mathrm{mHz}$, which might find correspondence in a slow-wave mode reflecting between the bow shock and the low-latitude boundary layer predicted by the model of Harrold and Samson (1992).

$b$ Nighttime average power spectra unambiguously show a greater relative importance of the low-frequency $H$ fluctuations between approximately 0.5 and $2 \mathrm{mHz}$ (30 and $10 \mathrm{~min}$ ) which can be related to the bay occurrence (Francia et al., 1995). Indeed, at low latitude, bays appear as gradual variations of the geomagnetic field components which typically persist for time-intervals ranging between several minutes and a few hours, and are currently interpreted as the low-latitude effects of the magnetosphericionospheric current systems developing at high latitude during substorms (Kamide, 1994, and references therein). In agreement with early observations (Chapman and Bartels, 1951), our results suggest that the geomagnetic-field variations related to the low-latitude bay occurrence mostly perturb the $H$ component, whose energy is on average larger than the energy of the $D$ fluctuations up to a factor of the order of $1.4-1.5$ between approximately 0.5 and $2 \mathrm{mHz}$. In this sense, our results can be considered qualitatively consistent with the three-dimensional current models (Kawasaki et al., 1974), which predict at low latitudes a much greater energy content in the $H$ component. Moreover, as suggested by previous investigations (Nishida, 1978) which underlined a clear tendency for low-latitude bays to occur slightly before midnight, we found (more explicitly at solar minimum) that $H$ perturbations become much more energetic in the premidnight sector and progressively disappear after local midnight; it would suggest that the central meridian of the substorm current wedge is on average located in the premidnight sector.

$c$ During nighttime intervals, the average power spectra do not show clear and simultaneous enhancements in the $H$ and $D$ power spectra. Rather, they show clear statistical evidence for a sharp $W$ enhancement at approximately $1.2 \mathrm{mHz}$, which might be related with magnetospheric perturbations predominantly occurring in the meridian plane. As for the bay occurrence, the $W$ peak emerges much more clearly in the premidnight sector at solar minimum. In this sense it is very interesting to underline that Samson et al. (1992) found that, at auroral latitudes, substorm intensifications were associated with resonant fluctuations of the geomagnetic field components at 1.3 and $1.9 \mathrm{mHz}$, and speculated that such field- line resonances, driven by cavity modes, might play an important role in the substorm process. In this context the results of the present investigation seem to suggest at least the mode occurring approximately at $1.2 \mathrm{mHz}$ as a feature which typically accompanies the low-latitude bay occurrence.

$d$ A comparison between observations performed at different solar-cycle phases appears to indicate that some aspects, such as the daytime simultaneous $H$ and $D$ enhancements, the fluctuation activity at periods smaller than $10 \mathrm{~min}$, the power level of the $H$ fluctuations, and the nighttime occurrence of $W$ peaks, appear more evident during conditions of higher solar-wind velocity. A preliminary analysis obtained during a two-month interval is very interesting in that it shows that these spectral features are highly amplified by extreme solar-wind velocities. In addition, we found that during this period the power enhancements emerge much more sharply at all the frequencies predicted by Harrold and Samson (1992). This is an interesting feature, in that in general, global modes are expected to be excited by several phenomena (such as pressure pulses from the solar wind and Kelvin-Helmoltz instability on the magnetopause) which are obviously related with large solar-wind velocities.

Acknowledgements. The authors are grateful to Prof. D. J. Southwood (Imperial College, London), J. C. Samson (University of Alberta, Edmonton), L. J. Lanzerotti (AT\&T Bell Laboratories), A. Wolfe (New York City Technical College) and to Dr. M. Vellante (University of L'Aquila) for helpful discussions. They also thank Dr. A. Meloni (Istituto Nazionale di Geofisica, Roma) who made available geomagnetic field observations from L'Aquila Geomagnetic Observatory. This research activity at L'Aquila is supported by MURST ( $40 \%$ and $60 \%$ contracts) and by GIFCO/CNR.

Topical Editor K.-H. Glaßmeier thanks C. Waters and S. Fujita for their help in evaluating this paper.

\section{References}

Chapman, S., and J. Bartels, Geomagnetism, vol. 1, Oxford Univ. Press, pp. 338, 1951.

Francia, P., U. Villante, and A. Meloni, An analysis of geomagnetic field variations $(3 \mathrm{~min}-2 \mathrm{~h})$ at a low-latitude observatory (L = 1.6), Ann. Geophysicae, 13, 522, 1995.

Harrold, B. G., and J. C. Samson, Standing ULF modes of the magnetosphere: a theory, Geophys. Res. Lett., 19, 1811, 1992.

Itonaga, M., and T. I. Kitamura, Effect of non-uniform ionospheric conductivity distributions on Pc3-5 magnetic pulsations - fast wave incidence, Ann. Geophysicae, 11, 366, 1993.

Kamide, Y., Ionospheric currents and magnetic disturbances at low latitudes during substorms, in Low-latitude ionospheric physics, Ed. Fu-Shong Kuo, Cospar Colloq. Ser. Vol. 7, pp. 251, 1994.

Kawasaki, K., C. I. Meng, and Y. Kamide, The development of three-dimensional current system during a magnetospheric substorm, Planet. Space Sci., 22, 1471, 1974.

Kivelson, M. G., Pulsations and magnetohydrodynamic waves, in Introduction to space physics, Eds. M. J. Kivelson and C. T. Russel, Cambridge University Press, pp. 330, 1995.

Kivelson, M. G., and D. J. Southwood, Resonant ULF waves: a new interpretation, Geophys. Res. Lett., 12, 49, 1985.

Kivelson, M. G., and D. J. Southwood, Hydromagnetic waves and the ionosphere, Geophys. Res. Lett., 15, 1271, 1988.

Meloni, A., F. Molina, P. Palangio, Q. Taccetti, and A. De Santis, Automatic digital recording of geomagnetic elements by means of a proton precession magnetometer, Geophys. Surv., 7, 339, 1984. 
Nishida, A., Geomagnetic diagnosis of the magnetosphere, Phy. Chem. Space, 9, 96, 1978.

Ruohoniemi, J. M., R. A. Greenwald, K. B. Baker, and J. C. Samson, HF radar observations of Pc5 field line resonances in the midnightearly morning MLT sector, J. Geophys. Res., 96, 15697, 1991.

Samson, J. C., and R. Rankin, The coupling of solar wind energy to MHD cavity modes, waveguide modes, and field line resonances in the Earth's magnetosphere, in Solar Wind Sources of Magnetospheric Ultra-Low-Frequency Waves, Geophys. Monogr., 81, AGU, 253, 1994.

Samson, J. C., D. D. Wallis, T. J. Hughes, F. Creutzberg, J. M. Ruohoniemi, and R. A. Greenwald, Substorm intensifications and field line resonances in the nightside magnetosphere, J. Geophys. Res., 97, 8495, 1992.
Vellante, M., and U. Villante, Maximum entropy spectral analysis of artificial sinusoidal signals, J. Geophys. Res., 89, 351, 1984.

Walker, A. D. M., J. M. Ruohoniemi, K. B. Baker, R. A. Greenwald, and J. C. Samson, Spatial and temporal behavior of ULF pulsations observed by the Goose Bay HF radar, J. Geophys. Res., 97, 12187, 1992.

Ziesolleck, C. W. S., and F. H. Chamalaun, A two-dimensional array study of low-latitude Pc5 geomagnetic pulsations, J. Geophys. Res., 98, 13703, 1993.

Ziesolleck, C. W. S., and D. R. McDiarmid, Auroral latitude Pc5 field line resonances: quantized frequencies, spatial characteristics, and diurnal variation, J. Geophys. Res., 99, 5817, 1994. 